\title{
Adaptive Backstepping Control of Nonlinear Hydraulic-Mechanical System Including Valve Dynamics
}

\author{
M. Choux G. Hovland \\ Mechatronics Group, Department of Engineering, University of Agder, N-4898 Grimstad, Norway. E-mail: \\ $\{$ martin.choux, geir.hovland\} @uia.no
}

\begin{abstract}
The main contribution of the paper is the development of an adaptive backstepping controller for a nonlinear hydraulic-mechanical system considering valve dynamics. The paper also compares the performance of two variants of an adaptive backstepping tracking controller with a simple PI controller. The results show that the backstepping controller considering valve dynamics achieves significantly better tracking performance than the PI controller, while handling uncertain parameters related to internal leakage, friction, the orifice equation and oil characteristics.
\end{abstract}

Keywords: adaptive observer backstepping, state feedback, nonlinear hydraulic-mechanical system, valve dynamics

\section{Introduction}

Control of nonlinear hydraulic-mechanical systems (NHMS) is challenging for several reasons: a) the system model is normally stiff with fast dynamics for the hydraulics and relatively slow dynamics for the mechanical parts, b) models usually contain strong nonlinear elements such as the flow in orifices, friction, valve overlap and input saturation, c) valves contain non-measurable states (position and velocity) and d) the oil characteristics depend on parameters such as temperature and air content.

Bonchis et al. (2002) present an experimental evaluation of ten different controller algorithms for an NHMS. The results in the paper show that the simple PI controller performs reasonably well, and only a few of the model-based controllers are able to improve the performance.

Adaptive backstepping is a model-based nonlinear control technique which has been recently applied to NHMS, see Zeng and Sepehri (2006, 2008). The back- stepping controller was not included in the survey of Bonchis et al. (2002). Hence, it would be of interest to compare the backstepping and the PI controller for an NHMS. In Zeng and Sepehri $(2006,2008)$ the authors presented an adaptive controller to handle internal leakage and unknown friction in a cylinder, unknown volumes in the orifice equation and temperature dependent oil characteristics.

One physical phenomenon not considered in Zeng and Sepehri $(2006,2008)$ is valve dynamics. Section 2.2 shows that valve dynamics can be significant and should be included in the model-based controller. In addition to the valve dynamics, the adaptive controller developed in this paper also handles internal leakage and unknown friction in the cylinder, unknown volumes in the orifice equation and temperature dependent oil characteristics.

Section 2 contains the model description including an experiment to determine the second order valve dynamics model, while sections 3 and 4 contain the controllers for two different scenarios: without and with 
valve dynamics while both controllers contain the nonlinear orifice equation. Section 5 contains simulation results with the two different backstepping controllers compared with a PI controller. Finally, section 6 contains the conclusions.

\section{Model Description}

The tracking of the mass position $y$ in the NHMS shown in Fig. 1 is considered.

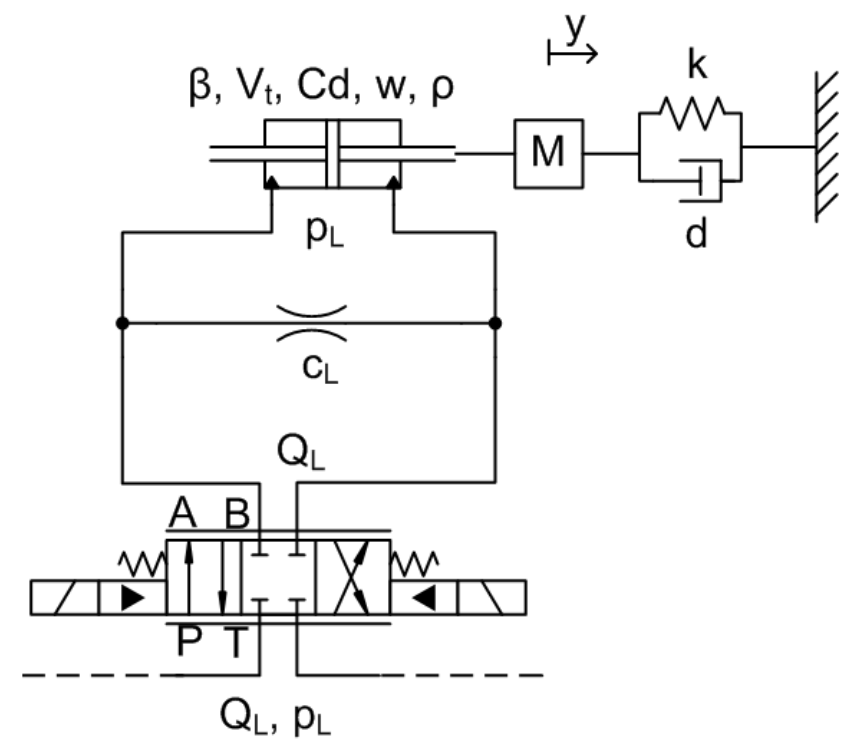

Figure 1: Translational hydraulic-mechanical system.

The parameters of the system are given in Table 1.

Table 1: Values of the system parameters with hydraulic units.

\begin{tabular}{clrl}
\hline Par. & Value & Par. & Value \\
\hline$M$ & $=41 \mathrm{~kg}$ & $K_{v}$ & $=1 \mathrm{~m} / \mathrm{A}$ \\
$k$ & $=65000 \mathrm{~N} / \mathrm{m}$ & $d$ & $=500 \mathrm{Ns} / \mathrm{m}$ \\
$A$ & $=946 \mathrm{~mm}^{2}$ & $\beta$ & $=12665 \mathrm{bar}$ \\
$\rho$ & $=900 \mathrm{~kg} / \mathrm{m}^{3}$ & $V_{t}$ & $=782 \mathrm{~cm}^{3}$ \\
$C_{d}$ & $=0.65$ & $w$ & $=7 \mathrm{~mm}$ \\
$p$ & $=80 \mathrm{bar}$ & $c_{L}$ & $=1$ \\
$\sigma_{0}$ & $=5880$ & $\sigma_{1}$ & $=108$ \\
$\sigma_{2}$ & $=500$ & $F_{c}$ & $=100$ \\
$F_{s}$ & $=200$ & $v_{s}$ & $=0.001$ \\
$Q_{L}$ & $=Q_{L} \mathrm{l} / \mathrm{min}$ & $y$ & $=y \mathrm{~m}$ \\
$p_{L}$ & $=p_{L} \mathrm{bar}$ & $x_{v}$ & $=x_{v} \mathrm{~mm}$ \\
$\omega_{v}$ & $=100 \mathrm{rad} / \mathrm{s}$ & $D$ & $=1$ \\
\hline
\end{tabular}

\subsection{Linear Friction Model}

In this work the influence of valve dynamics is the main focus of the paper. Extra states added by considering the dynamics of the friction model would complicate the study of the valve dynamics. In this regard the chosen friction model is linear:

$$
F_{\text {fric }}=\sigma \dot{y}
$$

The system in state space representation, with hydraulic units is:

$$
\begin{aligned}
\ddot{y} & =-\frac{k}{M} y-\frac{d+\sigma}{M} \dot{y}+\frac{A}{10 M} p_{L} \\
\dot{p}_{L} & =-\frac{4 \beta A}{V_{t}} \dot{y}-\frac{4 \beta}{V_{t}} c_{L} p_{L} \\
& +\frac{400 \sqrt{10} \beta C_{d} w K_{v}}{V_{t}} \sqrt{\frac{1}{\rho}} \sqrt{p-p_{L}} x_{v} \\
\ddot{x_{v}} & =-\omega_{v}^{2}\left(\frac{2 D}{\omega_{v}} \dot{x_{v}}+x_{v}\right)+K_{v} u
\end{aligned}
$$

where $u$ is the input current of the valve. If the state variables $\left[y, \dot{y}, p_{L}, x_{v}, \dot{x_{v}}\right]$ are equal to $\left[x_{1}, x_{2}, \frac{10 M x_{3}}{A}, x_{4}, x_{5}\right]$, the system can be rewritten as:

$$
\begin{aligned}
& \dot{x}_{1}=x_{2} \\
& \dot{x}_{2}=x_{3}+\varphi_{2}\left(x_{1}, x_{2}\right)^{T} \theta \\
& \dot{x}_{3}=b f\left(x_{3}\right) x_{4}+\varphi_{3}\left(x_{2}, x_{3}\right)^{T} \theta \\
& \dot{x}_{4}=x_{5} \\
& \dot{x}_{5}=\varphi_{5}\left(x_{4}, x_{5}\right)^{T} \theta+u
\end{aligned}
$$

where $\theta$ is the vector of unknown parameters:

$$
\begin{gathered}
\theta=\left[\theta_{1}, \cdots, \theta_{6}\right]^{T}=\left[\frac{-k}{M}, \frac{-d+\sigma}{M},\right. \\
\left.\frac{-2 \beta A^{2}}{5 M V_{t}}, \frac{-4 \beta c_{L}}{V_{t}},-\omega_{v}^{2},-2 D \omega_{v}\right]^{T}
\end{gathered}
$$

$b$ is a known-scalar:

$$
b=\frac{40 \sqrt{10} A \beta C_{d} \omega K_{v}}{M V_{t}} \sqrt{\frac{1}{\rho}}
$$

and $f$ is a nonlinear function:

$$
f\left(p_{L}\right)=\sqrt{p-\operatorname{sign}\left(x_{v}\right) p_{L}}
$$

The vector functions $\varphi_{k}(k \in\{2,3,5\})$ are defined as: $\varphi_{2}\left(x_{1}, x_{2}\right)=\left[x_{1}, x_{2}, 0,0,0,0\right]^{T}, \varphi_{3}\left(x_{2}, x_{3}\right)=$ $\left[0,0, x_{2}, x_{3}, 0,0\right]^{T}$ and $\varphi_{5}\left(x_{4}, x_{5}\right)=\left[0,0,0,0, x_{4}, x_{5}\right]^{T}$. 


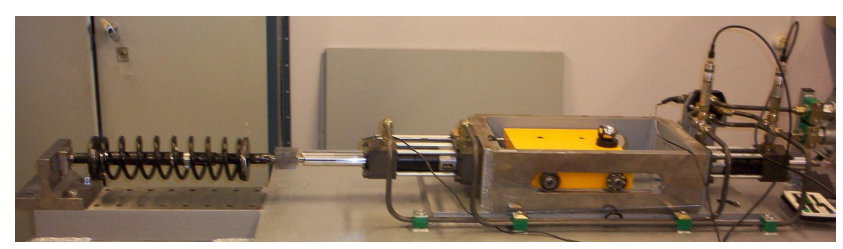

Figure 2: Experimental setup for NHMS.

\subsection{Valve Dynamics}

An experimental setup shown in Fig. 2 has been used to determine the valve dynamics. A proportional and a second order valve dynamics model are compared. The proportional model is given by $x_{v}=K_{v} u$ while the second order model is given by eq. (4). A step response was generated by using a PI controller on the experimental setup in Fig. 2 and also on two simulated models with: a) proportional valve characteristics and b) a second order valve dynamics model (eq. (4)). The results are shown in Figs. 3 and 4. Fig. 3 shows the entire step response and the reverse step response, while Fig. 4 is zoomed in at the transient response where the errors are the largest.

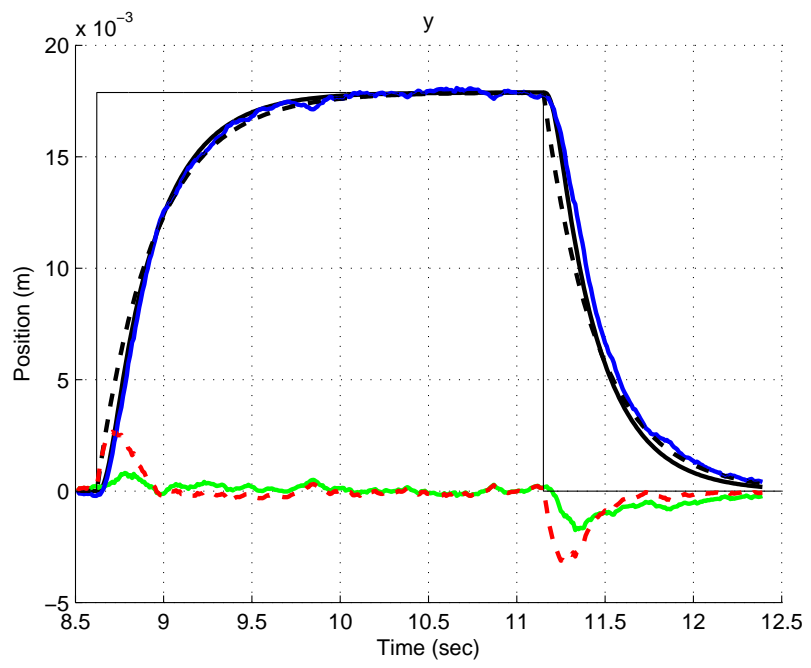

Figure 3: Experimental and simulated results with two different valve models. Blue: measurements, Black: second order model, Green: error second order model, Dashed: proportional model, Red: error proportional model.

Table 2 shows that the second order model represents a significant improvement compared to the proportional model. The step response (position) is improved 52\% and $73 \%$, respectively, for the RMS and MAX values. Table 2 also shows the RMS and MAX values for the

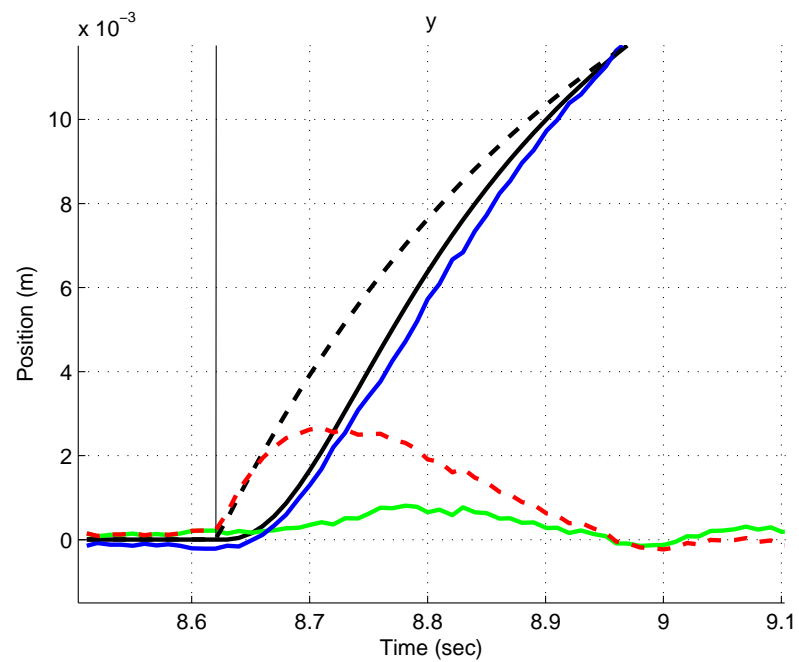

Figure 4: Transient response. Blue: measurements, Black: second order model, Green: error second order model, Dashed: proportional model, Red: error proportional model.

\begin{tabular}{|c|c|c|c|}
\hline Position & Proportional & $2^{\text {nd }}$ order & Improvement \\
\hline RMS & $7.16 \cdot 10^{-4}$ & $3.41 \cdot 10^{-4}$ & $52 \%$ \\
\hline $\mid$ MAX & $9.19 \cdot 10^{-6}$ & $2.46 \cdot 10^{-6}$ & $73 \%$ \\
\hline Pressure & Proportional & $2^{\text {nd }}$ order & Improvement \\
\hline RMS & 1.94 & 1.96 & $-1 \%$ \\
\hline$|\mathrm{MAX}|$ & 98.1 & 46.6 & $110 \%$ \\
\hline
\end{tabular}

Table 2: Comparison of RMS and MAX values against experiments for a step response using a proportional and a second order valve dynamics model. Top: Position, Bottom: Pressure.

measured load pressure $p_{L}$ vs. the simulated load pressures. The pressure RMS values for the proportional and second order model are similar, while the MAX value shows a significant improvement for the second order model. Hence, the effects of the valve dynamics are important to consider in high-performance control of NHMS.

\section{Backstepping without Valve Dynamics}

In order to demonstrate the effects of introducing valve dynamics in the backstepping controller, a controller without considering valve dynamics is developed first. The complete controller including valve dynamics is presented in section 4.

The system without valve dynamics can be rewritten 
as:

$$
\begin{gathered}
\ddot{y}=-\frac{k}{M} y-\frac{d+\sigma_{2}}{M} \dot{y}+\frac{A}{10 M} p_{L} \\
\frac{A}{10 M} \dot{p}_{L}=-\frac{2 \beta A^{2}}{5 M V_{t}} \dot{y}-\frac{4 \beta}{V_{t}} c_{L} \frac{A}{10 M} p_{L} \\
+\frac{40 \sqrt{10} A \beta C_{d} w K_{v}}{M V_{t} \sqrt{\rho}} \sqrt{p-\operatorname{sign}(u) \frac{10 M}{A} \frac{A}{10 M} p_{L} u}
\end{gathered}
$$

or, alternatively

$$
\begin{aligned}
& \dot{x}_{1}=x_{2} \\
& \dot{x}_{2}=x_{3}+\varphi_{2}\left(x_{1}, x_{2}\right)^{T} \theta \\
& \dot{x}_{3}=b f\left(x_{3}\right) u+\varphi_{3}\left(x_{2}, x_{3}\right)^{T} \theta
\end{aligned}
$$

where $\theta$ and $\varphi$ are reduced by two states, ie. $\theta=\left[\theta_{1}, \cdots, \theta_{4}\right]^{T}=\left[\frac{-k}{M}, \frac{-(d+\sigma)}{M}, \frac{-2 \beta A^{2}}{5 M V_{t}}, \frac{-4 \beta c_{L}}{V_{t}}\right]^{T}$, $\varphi_{2}\left(x_{1}, x_{2}\right)=\left[x_{1}, x_{2}, 0,0\right]^{T}, \varphi_{3}\left(x_{2}, x_{3}\right)=\left[0,0, x_{2}, x_{3}\right]^{T}$ and $f\left(x_{3}\right)=\sqrt{p-\operatorname{sign}(u) \frac{10 M}{A} x_{3}}$. Following the tuning function design as in Krstić et al. (1995), the state space system (15-17), which is in a strict feedback form can be decomposed in sucessive subsystems for which tuning functions and stabilizing functions are recursively found, leading to the final adaptive control law $u$ and the final update law for the uncertain parameters $\theta$ and $\lambda=\frac{1}{b}$ with estimated $\hat{\theta}$ and $\hat{\lambda}$. Note that the symbol $\sim$ defines the estimation error, ie. $\tilde{\theta}=\theta-\hat{\theta}$.

\section{Coordinate Transformation}

$$
\begin{aligned}
& z_{1}=x_{1}-y_{r} \\
& z_{2}=x_{2}-y_{r}^{(1)}-\alpha_{1} \\
& z_{3}=x_{3}-y_{r}^{(2)}-\alpha_{2}
\end{aligned}
$$

\section{Regressor}

$$
\begin{aligned}
\omega_{1} & =0 \\
\omega_{2} & =\phi_{2} \\
\omega_{3} & =\phi_{3}-\frac{\partial \alpha_{2}}{\partial x_{2}} \phi_{2}
\end{aligned}
$$

\section{Tuning functions for $\hat{\theta}$ :}

$$
\begin{aligned}
\tau_{1} & =0 \\
\tau_{2} & =\omega_{2} z_{2} \\
\tau_{3} & =\tau_{2}+\omega_{3} z_{3}
\end{aligned}
$$

\section{Stabilizing functions:}

$$
\begin{aligned}
\alpha_{1}\left(x_{1}, y_{r}\right) & =\bar{\alpha}_{1} \\
\alpha_{2}\left(x_{1}, x_{2}, \hat{\theta}, y_{r}, \dot{y}_{r}\right) & =\bar{\alpha}_{2} \\
\alpha_{3}\left(\bar{x}_{3}, \hat{\theta}, \bar{y}_{r}^{(2)}, \hat{\lambda}\right) & =\frac{\hat{\lambda}}{f\left(x_{3}\right)} \bar{\alpha}_{3}
\end{aligned}
$$

$$
\bar{\alpha}_{1}=-L_{1} z_{1}
$$

$$
\begin{aligned}
\bar{\alpha}_{2} & =-z_{1}-L_{2} z_{2}-\omega_{2}^{T} \hat{\theta}+\frac{\partial \alpha_{1}}{\partial x_{1}} x_{2} \\
& +\frac{\partial \alpha_{1}}{\partial y_{r}} \dot{y}_{r}
\end{aligned}
$$

$$
\begin{aligned}
\bar{\alpha}_{3} & =-z_{2}-L_{3} z_{3}-\omega_{3}^{T} \hat{\theta}+\frac{\partial \alpha_{2}}{\partial \hat{\theta}} \Gamma \tau_{3} \\
& +\sum_{k=1}^{2}\left(\frac{\partial \alpha_{2}}{\partial x_{k}} x_{k+1}+\frac{\partial \alpha_{2}}{\partial y_{r}^{(k-1)}} y_{r}^{(k)}\right)
\end{aligned}
$$

\section{Adaptive control law:}

$$
u=\alpha_{3}+\frac{\hat{\lambda}}{f\left(x_{3}\right)} y_{r}^{(3)}
$$

\section{Parameter update laws:}

$$
\begin{aligned}
& \dot{\hat{\theta}}=\Gamma \tau_{3} \\
& \dot{\hat{\lambda}}=-\gamma \operatorname{sign}(b)\left(y_{r}^{(3)}+\bar{\alpha}_{3}\right) z_{3}
\end{aligned}
$$

Error system: The design procedure (18-35) results in the following error system:

$$
\begin{aligned}
& \dot{z}_{1}=-L_{1} z_{1}+z_{2} \\
& \dot{z}_{2}=-L_{2} z_{2}-z_{1}+z_{3}+\omega_{2}^{T} \tilde{\theta} \\
& \dot{z}_{3}=-L_{3} z_{3}-z_{2}+\omega_{3}^{T} \tilde{\theta}-b\left(\bar{\alpha}_{3}+y_{r}^{(3)}\right) \tilde{\lambda}
\end{aligned}
$$

A Lyapunov function for this system is:

$$
V=\frac{1}{2} z^{T} z+\frac{1}{2} \tilde{\theta}^{T} \Gamma^{-1} \tilde{\theta}+\frac{|b|}{2 \gamma} \tilde{\lambda}^{2}
$$

Its derivative along the solution of eqs. (34-35) and (36-38) is:

$$
\dot{V}=-\sum_{k=1}^{5} L_{k} z_{k}^{2}
$$

which proves from the Lasalle-Yoshizawa theorem that global asymptotic tracking is achieved. The calculations for the error system and the Lyapunov derivation are not shown in this paper, but are similar to the more complicated calculations for the controller including valve dynamics in Appendices A and B. 


\section{Backstepping with Valve Dynamics}

In this subsection valve dynamics is included and it is assumed that all the states, i.e. position and velocity of the mass, load pressure, position and velocity of the valve spool, are measured. The system rewritten as:

$$
\begin{aligned}
& \dot{x}_{1}=x_{2} \\
& \dot{x}_{2}=x_{3}+\varphi_{2}\left(x_{1}, x_{2}\right)^{T} \theta \\
& \dot{x}_{3}=b f\left(x_{3}\right) x_{4}+\varphi_{3}\left(x_{2}, x_{3}\right)^{T} \theta \\
& \dot{x}_{4}=x_{5} \\
& \dot{x}_{5}=u+\varphi_{5}\left(x_{4}, x_{5}\right)^{T} \theta
\end{aligned}
$$

is in strict-feedback form but contains an unknown virtual control coefficient $b f\left(x_{3}\right)$ which is not constant. A new extension of the tuning function design from Krstić et al. (1995) is developed below in the special case where $f(x)=\sqrt{p-x}$ :

\section{Coordinate Transformation}

$$
\begin{aligned}
& z_{1}=x_{1}-y_{r} \\
& z_{2}=x_{2}-y_{r}^{(1)}-\alpha_{1} \\
& z_{3}=x_{3}-y_{r}^{(2)}-\alpha_{2} \\
& z_{4}=x_{4}-\frac{\hat{\lambda}}{f\left(x_{3}\right)} y_{r}^{(3)}-\alpha_{3} \\
& z_{5}=x_{5}-\frac{\hat{\lambda}}{f\left(x_{3}\right)} y_{r}^{(4)}-\alpha_{4}
\end{aligned}
$$

\section{Regressor}

$$
\begin{aligned}
& \omega_{1}=0 \\
& \omega_{2}=\phi_{2} \\
& \omega_{3}=\phi_{3}-\frac{\partial \alpha_{2}}{\partial x_{2}} \phi_{2} \\
& \omega_{4}=-\frac{\partial \alpha_{3}}{\partial x_{2}} \phi_{2}-\frac{\partial \alpha_{3}}{\partial x_{3}} \phi_{3}+\frac{\hat{\lambda} y_{r}^{(3)}}{2 f\left(x_{3}\right)^{3}} \phi_{3} \\
& \omega_{5}=\phi_{5}-\frac{\partial \alpha_{4}}{\partial x_{2}} \phi_{2}-\frac{\partial \alpha_{4}}{\partial x_{3}} \phi_{3}+\frac{\hat{\lambda} y_{r}^{(4)}}{2 f\left(x_{3}\right)^{3}} \phi_{3}
\end{aligned}
$$

\section{Tuning functions for $\hat{\theta}$ :}

$$
\begin{aligned}
\tau_{1} & =0 \\
\tau_{2} & =\omega_{2} z_{2} \\
\tau_{3} & =\tau_{2}+\omega_{3} z_{3} \\
\tau_{4} & =\tau_{3}+\omega_{4} z_{4} \\
\tau_{5} & =\tau_{4}+\omega_{5} z_{5}
\end{aligned}
$$

\section{Stabilizing functions:}

$$
\begin{aligned}
\alpha_{1}\left(x_{1}, y_{r}\right) & =\bar{\alpha}_{1} \\
\alpha_{2}\left(x_{1}, x_{2}, \hat{\theta}, y_{r}, \dot{y}_{r}\right) & =\bar{\alpha}_{2} \\
\alpha_{3}\left(\bar{x}_{3}, \hat{\theta}, \bar{y}_{r}^{(2)}, \hat{\lambda}\right) & =\frac{\hat{\lambda}}{f\left(x_{3}\right)} \bar{\alpha}_{3} \\
\alpha_{4}\left(\bar{x}_{4}, \hat{\theta}, \bar{y}_{r}^{(3)}, \hat{b}, \hat{\lambda}\right) & =\bar{\alpha}_{4} \\
\alpha_{5}\left(\bar{x}_{5}, \hat{\theta}, \bar{y}_{r}^{(4)}, \hat{b}, \hat{\lambda}\right) & =\bar{\alpha}_{5}
\end{aligned}
$$

$$
\begin{aligned}
\bar{\alpha}_{4} & =-\hat{b} f\left(x_{3}\right) z_{3}-L_{4} z_{4}-\omega_{4}^{T} \hat{\theta}+\frac{\partial \alpha_{3}}{\partial \hat{\theta}} \Gamma \tau_{4} \\
& +\sum_{k=1}^{2} \frac{\partial \alpha_{3}}{\partial x_{k}} x_{k+1}+f\left(x_{3}\right) \hat{b} \frac{\partial \alpha_{3}}{\partial x_{3}} x_{4}+\sum_{k=1}^{3} \frac{\partial \alpha_{3}}{\partial y_{r}^{(k-1)}} y_{r}^{(k)}
\end{aligned}
$$$$
+\left(\frac{y_{r}^{(3)}}{f\left(x_{3}\right)}+\frac{\partial \alpha_{3}}{\partial \hat{\lambda}}\right) \dot{\hat{\lambda}}+\sum_{k=2}^{3} \frac{\partial \alpha_{k-1}}{\partial \hat{\theta}} \Gamma \omega_{4} z_{k}
$$$$
-\frac{\hat{\lambda} y_{r}^{(3)} \hat{b}}{2 f\left(x_{3}\right)^{2}} x_{4}
$$

$\bar{\alpha}_{5}=-z_{4}-L_{5} z_{5}-\omega_{5}^{T} \hat{\theta}+\frac{\partial \alpha_{4}}{\partial \hat{\theta}} \Gamma \tau_{5}$

$$
\begin{aligned}
& +\sum_{\substack{k=1 \\
k \neq 3}}^{4} \frac{\partial \alpha_{4}}{\partial x_{k}} x_{k+1}+f\left(x_{3}\right) \hat{b} \frac{\partial \alpha_{4}}{\partial x_{3}} x_{4}+\sum_{k=1}^{4} \frac{\partial \alpha_{4}}{\partial y_{r}^{(k-1)}} y_{r}^{(k)} \\
& +\left(\frac{y_{r}^{(4)}}{f\left(x_{3}\right)}+\frac{\partial \alpha_{4}}{\partial \hat{\lambda}}\right) \dot{\hat{\lambda}}+\sum_{k=2}^{4} \frac{\partial \alpha_{k-1}}{\partial \hat{\theta}} \Gamma \omega_{5} z_{k} \\
& +\frac{\partial \alpha 4}{\partial \hat{b}} \gamma \Pi_{5}-\frac{\hat{\lambda} y_{r}^{(4)} \hat{b}}{2 f\left(x_{3}\right)^{2}} x_{4}
\end{aligned}
$$




\section{Adaptive control law:}

$$
u=\alpha_{5}+\frac{\hat{\lambda}}{f\left(x_{3}\right)} y_{r}^{(5)}
$$

\section{Parameter update laws:}

$$
\begin{aligned}
& \dot{\hat{\theta}}=\Gamma \tau_{5} \\
& \dot{\hat{b}}=\gamma \pi_{5} \\
& \dot{\hat{\lambda}}=-\gamma \operatorname{sign}(b)\left(y_{r}^{(3)}+\bar{\alpha}_{3}\right) z_{3}
\end{aligned}
$$

Error system: The design procedure (46-77) results in the following error system (See Appendix A):

$$
\begin{aligned}
\dot{z}_{1} & =-L_{1} z_{1}+z_{2} \\
\dot{z}_{2} & =-L_{2} z_{2}-z_{1}+z_{3}+\omega_{2}^{T} \tilde{\theta} \\
\dot{z}_{3} & =-L_{3} z_{3}-z_{2}+\hat{b} f\left(x_{3}\right) z_{4}+\sigma_{34} z_{4}+\sigma_{35} z_{5} \\
& +\omega_{3}^{T} \tilde{\theta}-b\left(\bar{\alpha}_{3}+y_{r}^{(3)}\right) \tilde{\lambda}+\tilde{b} f\left(x_{3}\right) z_{4}
\end{aligned}
$$

$$
\begin{aligned}
\dot{z}_{4} & =-L_{4} z_{4}-\sigma_{34} z_{3}-\hat{b} f\left(x_{3}\right) z_{3}+z_{5}+\sigma_{55} z_{5} \\
& +\omega_{4}^{T} \tilde{\theta}+\tilde{b}\left(\frac{\hat{\lambda} y_{r}^{(3)}}{2 f\left(x_{3}\right)^{2}} x_{4}-f\left(x_{3}\right) \frac{\partial \alpha_{3}}{\partial x_{3}} x_{4}\right) \\
\dot{z}_{5} & =-L_{5} z_{5}-\sigma_{35} z_{3}-\sigma_{45} z_{4}-z_{4} \\
& +\omega_{5}^{T} \tilde{\theta}+\tilde{b}\left(\frac{\hat{\lambda} y_{r}^{(4)}}{2 f\left(x_{3}\right)^{2}} x_{4}-f\left(x_{3}\right) \frac{\partial \alpha_{4}}{\partial x_{3}} x_{4}\right)
\end{aligned}
$$

where $\sigma_{i k}$ is defined as

$$
\begin{aligned}
\sigma_{34} & =-\frac{\partial \alpha_{2}}{\partial \hat{\theta}} \Gamma \omega_{4} \\
\sigma_{35} & =-\frac{\partial \alpha_{2}}{\partial \hat{\theta}} \Gamma \omega_{5} \\
\sigma_{45} & =-\frac{\partial \alpha_{3}}{\partial \hat{\theta}} \Gamma \omega_{5}
\end{aligned}
$$

A Lyapunov function for this system is:

$$
V=\frac{1}{2} z^{T} z+\frac{1}{2} \tilde{\theta}^{T} \Gamma^{-1} \tilde{\theta}+\frac{1}{2 \gamma} \tilde{b}^{2}+\frac{|b|}{2 \gamma} \tilde{\lambda}^{2}
$$

Its derivative along the solutions of (78-85) is (See Appendix B):

$$
\dot{V}=-\sum_{k=1}^{5} L_{k} z_{k}^{2}
$$

\begin{tabular}{|l|l|l|}
\hline Cost & Original & Optimized \\
\hline$\alpha_{1}$ & $1 \otimes 2 \oplus$ & $1 \otimes 2 \oplus$ \\
\hline$\alpha_{2}$ & $6 \otimes 9 \oplus$ & $6 \otimes 9 \oplus$ \\
\hline$\alpha_{3}$ & $43 \otimes 67 \oplus$ & $27 \otimes 30 \oplus 7 \triangleright$ \\
\hline$\alpha_{4}$ & $707 \otimes 1085 \oplus$ & $128 \otimes 122 \oplus 53 \triangleright$ \\
\hline$\alpha_{5}$ & $29591 \otimes 44486 \oplus$ & $699 \otimes 513 \oplus 222 \triangleright$ \\
\hline
\end{tabular}

Table 3: Cost of calculation in number of multiplication $(\otimes)$, number of additions $(\oplus)$ and number of assignments ( $\triangleright$ ) for each stabilizing function $\alpha_{i}$. Last column is the cost when the calculations are optimized.

Table 3 shows the cost of calculations for each stabilizing function at each design step. The computation of the final control law is optimized in order to reduce the cost of calculation and make real time application possible. Table 3 shows that significant reduction in calculation time is possible by optimizing the code.

\section{Simulations}

\subsection{Tracking Performance of Backstepping Controllers}

In order to test the robustness of the controller, two models of the plant are implemented. The first one, described in section 3 , is used to design the controller, whereas a second model, more realistic is used to represent the physical system. In this new model the dynamics of the valve is represented by a second order transfer function, the friction in the cylinder is nonlinear and Stribeck and Coulomb effects are modeled. Moreover the compressibility of the fluid is not neglected inside the load and thus can the cylinder accumulate fluid. Finally the uncertain parameters of the new model differ from the ones used in the controller design by up to $+/-20 \%$. The simulation results are given in Fig. 5-10. Fig. 5 and 8 show the tracking for sinusoidal and step references. Fig. 6 and 9 shows the tracking error, and Fig. 7 and 10 show the actuator (valve) input. For the Figs. 5-7 the controller gains equal $\left[L_{1}, L_{2}, L_{3}\right]=[180,180,180]$, while for Figs. 810 the controller gains equal $\left[L_{1}, L_{2}, L_{3}, L_{4}, L_{5}\right]=$ $[180,180,180,350,350]$. The reference position and the tracking position are shown with dashed and plain lines, respectively. The model used to develop both the backstepping controllers contain the following uncertainties: $M^{*}=0.9 \mathrm{M}, A^{*}=1.1 \mathrm{~A}, \mathrm{k}^{*}=0.8 \mathrm{k}$, $d^{*}=0.8 d, C_{d}^{*}=0.9 C_{d}, w^{*}=1.1 \mathrm{w}, V_{t}^{*}=0.8 V_{t}$, $\beta^{*}=0.8 \beta, \rho^{*}=0.9 \rho, p^{*}=0.9 p$. The $*$-superscript refers to the model used by the controller. 


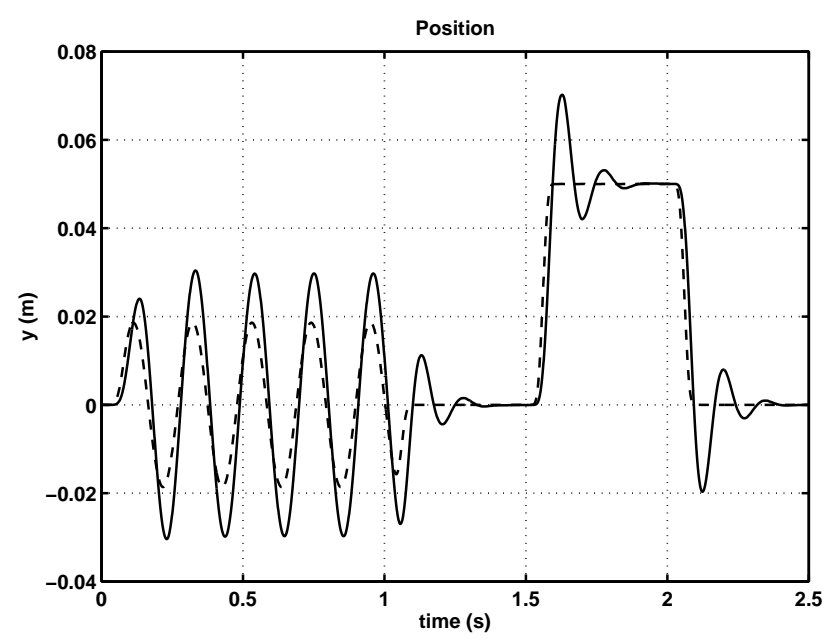

Figure 5: Position tracking with the controller of section 3 .

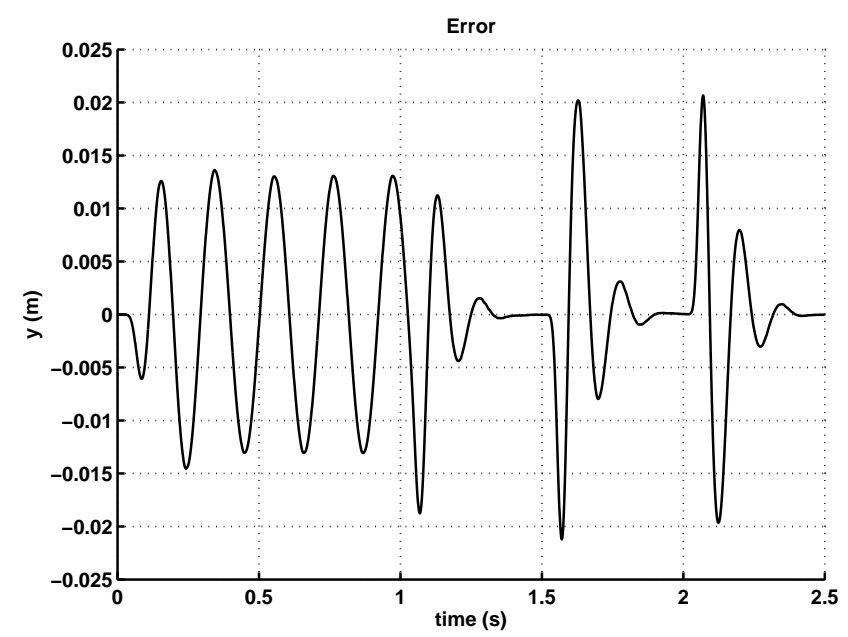

Figure 6: Tracking error for Fig. 5.

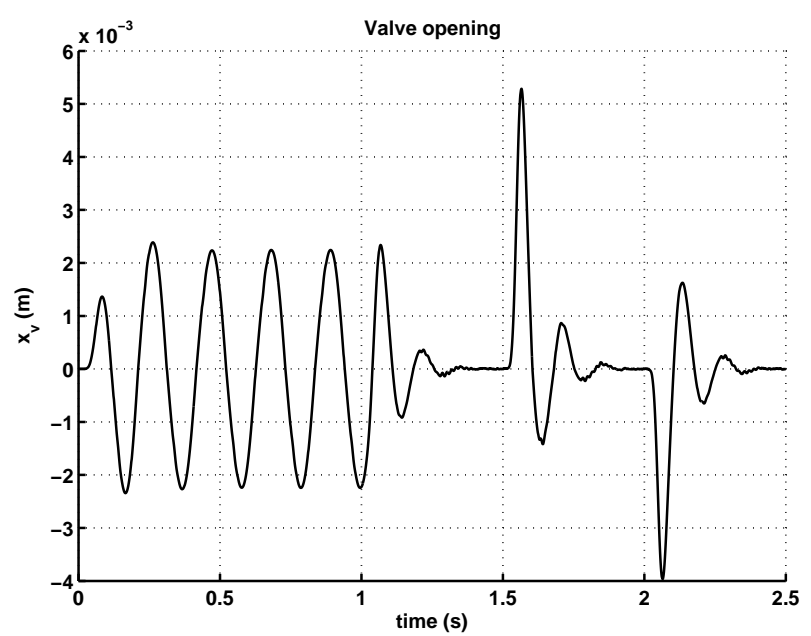

Figure 7: Input (valve opening) with the controller of section 3 .

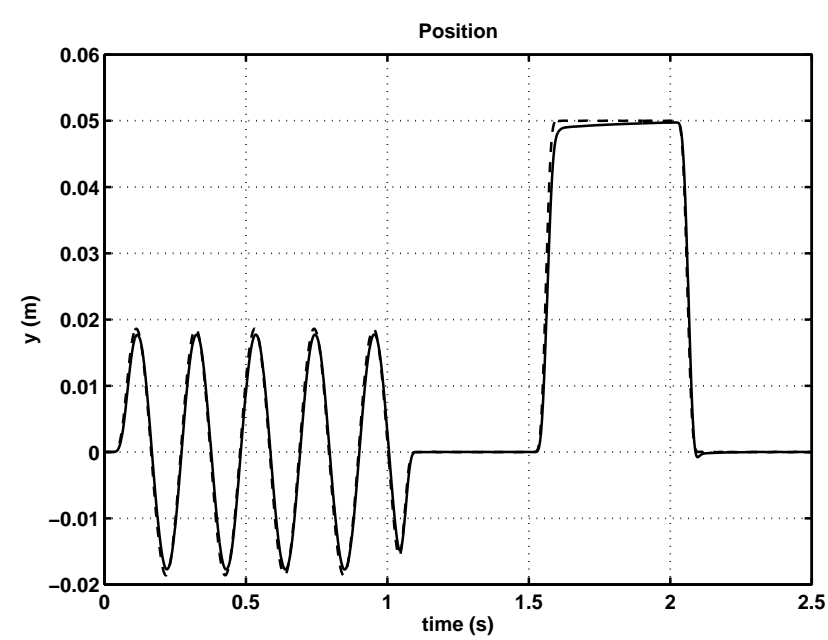

Figure 8: Position tracking with the controller of section 4.

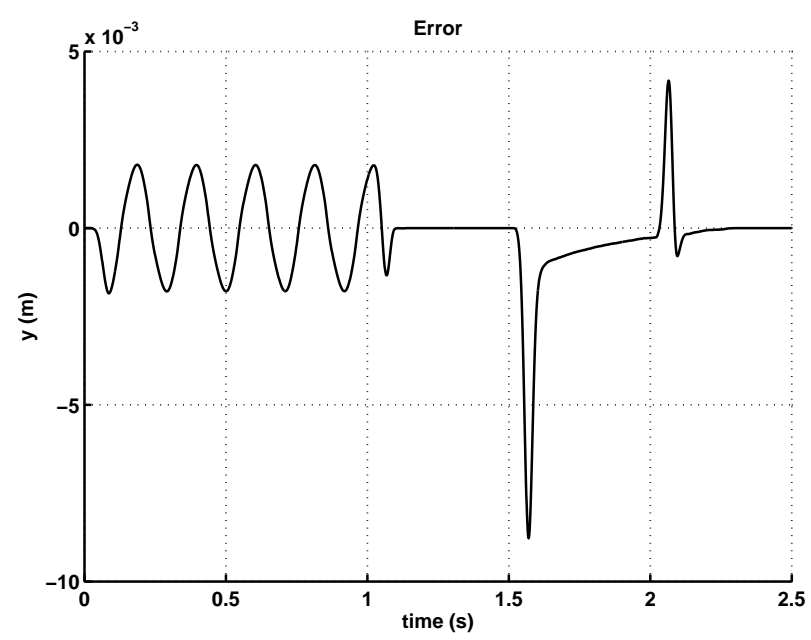

Figure 9: Tracking error for Fig. 8.

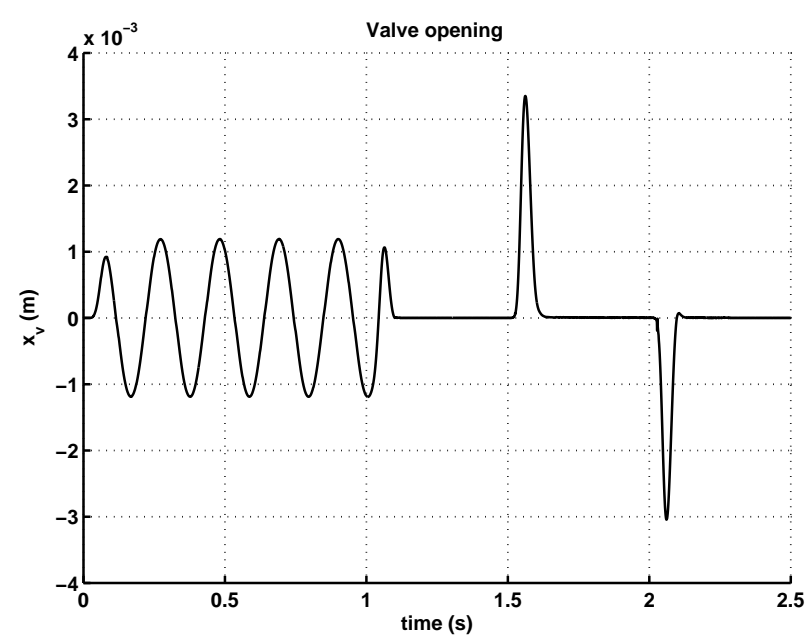

Figure 10: Input (valve opening) with the controller of section 4 . 


\subsection{Comparison with PI Controller}

In Bonchis et al. (2002) the following comparison criteria were defined: Mean Positioning Accuracy (MPA), Absolute Positioning Accuracy (APA), Weighted Position Accuracy (WPA), Saturation Index (SAT), Robustness Index (RI) and Composite Index (CI). A PD controller was compared with the other controllers on all these criteria for the transient response $\left(t_{s}=0\right)$ and the steady-state performance $\left(t_{s}=10\right)$, as well as for a sinusoidal response and for a point-to-point response. For the RI Bonchis et al. (2002) used a 50\% reduction in supply pressure.

In this paper, the criteria APA, MPA and WPA are used to compare a PI controller with two backstepping controllers BS1 and BS2 of sections 3 and 4, respectively. A PI controller is used instead of a PD, because the spring in Fig. 1 makes the open-loop integrator disappear. The hydraulic system considered in Bonchis et al. (2002) contained an open-loop integrator. Moreover, the following three criteria are not considered in this paper: SAT, RI and CI. Input saturation for the system in Fig. 1 occurs when the valve opening reaches $5 \mathrm{~mm}$. This saturation only occurs for the controller in section 3. The criterion RI is not suited to benchmarking when the nominal error is close to zero, which is the case in this paper. The CI makes use of the RI, and hence is also not suited in our case. Nevertheless, the robustness of the adaptive backstepping controller can be seen for example in Fig. 8, where a $20 \%$ initial error in model parameters are introduced.

Similar to the presentation in Bonchis et al. (2002), Figs. 11-13 contain 4 bars, representing a) sinusoidal reference (entire response), b) sinusoidal reference (steady-state response), c) point-to-point reference (entire response), d) point-to-point (steady-state). Fig. 11 shows that both backstepping controllers BS1 and BS2 perform better than the PI controller for the APA criterion, where the BS2 controller performs significantly better. For both the MPA and the WPA, the BS1 and the PI controllers give similar performance, while the BS2 performs significantly better as seen in Figs. 12-13.

\section{Conclusions}

In this paper an adaptive backstepping controller considering valve dynamics for a nonlinear hydraulicmechanical system has been developed and the performance has been compared with three different criteria to a simple PI controller. All three criteria show that the adaptive backstepping controller taking valve dynamics into account performs significantly better than both the PI controller and a reduced version of the

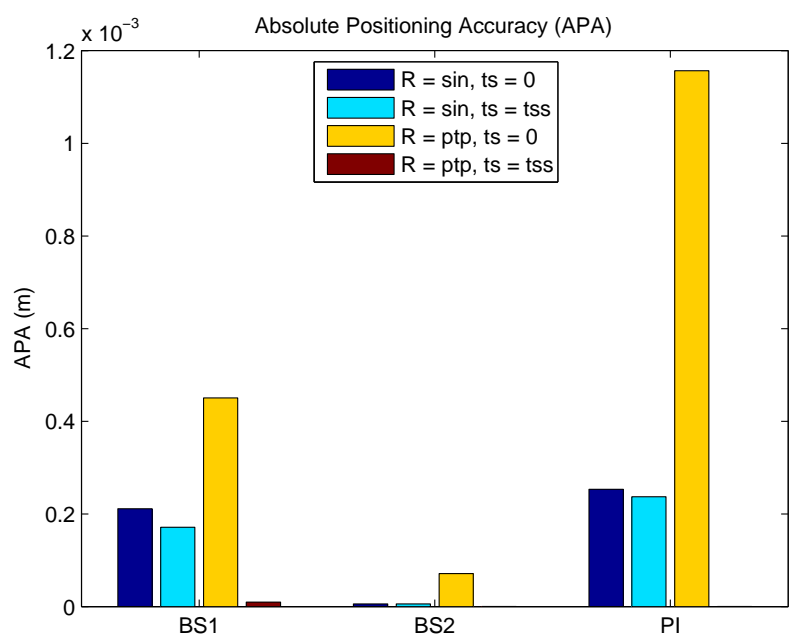

Figure 11: Comparison of Absolute Positioning Accuracy.

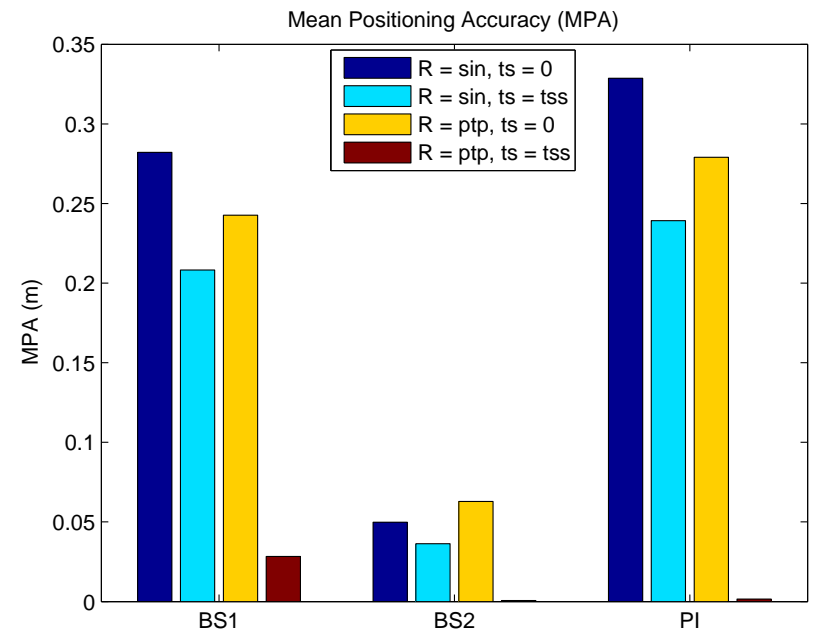

Figure 12: Comparison of Mean Positioning Accuracy.

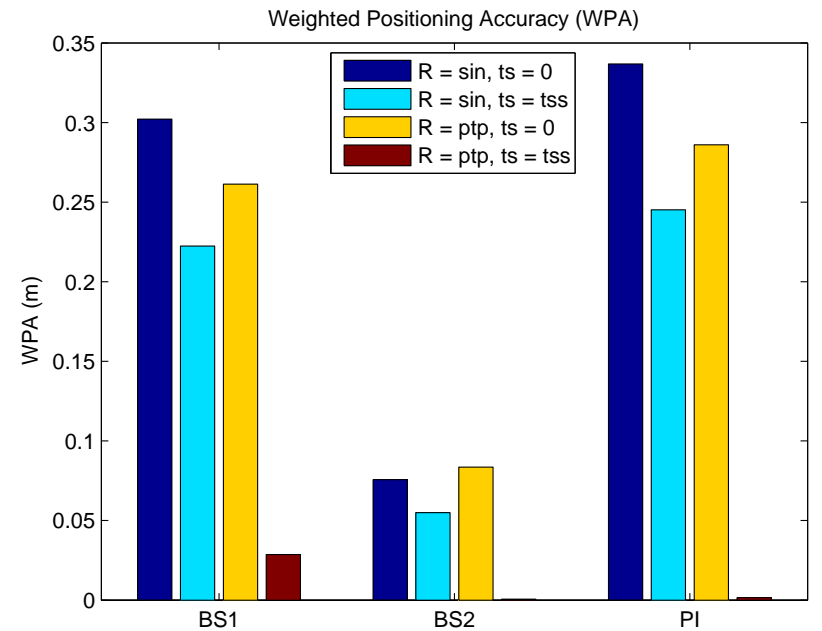

Figure 13: Comparison of Weighted Positioning Accuracy. 
backstepping controller without taking valve dynamics into account.

Future research directions will focus on developing an output-feedback version of the backstepping controller and the implementation of this controller on the experimental setup shown in Fig. 2.

\section{References}

Bonchis, A., Corke, P., and Rye, D. Experimental Evaluation of Position Control Methods for Hydraulic Systems. IEEE Transactions on Control Systems Technology, 2002. 10(6):876-882. doi:10.1109/TCST.2002.804128.

Krstić, M., Kanellakopoulos, I., and Kokotović, P. Nonlinear and Adaptive Control Design. Wiley, New York, 1995.

Zeng, H. and Sepehri, N. Adaptive backstepping control of hydraulic manipulators with friction compensation using LuGre model. In Proc. American Control Conference. pages 3164-3169, 2006.

Zeng, H. and Sepehri, N. Tracking Control of Hydraulic Actuators Using a LuGre Friction Model Compensation. Journal of Dynamic Systems, Measurement, and Control, 2008. 130(1):0145021-0145027. doi:10.1115/1.2807181.

\section{A. Calculations for the Error System Including Valve Dynamics}

$$
\begin{aligned}
\dot{z}_{1} & =\dot{x}_{1}-y_{r}^{(1)} \\
& =x_{2}-y_{r}^{(1)} \\
& =z_{2}+\alpha_{1} \\
& =-L_{1} z_{1}+z_{2} \\
\dot{z}_{2} & =\dot{x}_{2}-y_{r}^{(2)}-\dot{\alpha}_{1} \\
& =x_{3}+\varphi_{2}\left(x_{1}, x_{2}\right)^{T} \hat{\theta}+\varphi_{2}\left(x_{1}, x_{2}\right)^{T} \tilde{\theta}-y_{r}^{(2)}-\dot{\alpha}_{1} \\
& =z_{3}+\varphi_{2}\left(x_{1}, x_{2}\right)^{T} \hat{\theta}+\varphi_{2}\left(x_{1}, x_{2}\right)^{T} \tilde{\theta}-\dot{\alpha}_{1}+\alpha_{2} \\
& =z_{3}+\varphi_{2}\left(x_{1}, x_{2}\right)^{T} \hat{\theta}+\varphi_{2}\left(x_{1}, x_{2}\right)^{T} \tilde{\theta}-\dot{\alpha}_{1} \\
& -z_{1}-L_{2} z_{2}-\omega_{2}^{T} \hat{\theta}+\frac{\partial \alpha_{1}}{\partial x_{1}} x_{2}+\frac{\partial \alpha_{1}}{\partial y_{r}} \dot{y}_{r} \\
& =-L_{2} z_{2}-z_{1}+z_{3}+\omega_{2}^{T} \tilde{\theta}
\end{aligned}
$$

$$
\begin{aligned}
\dot{z}_{3} & =\dot{x}_{3}-y_{r}^{(3)}-\dot{\alpha}_{2} \\
& =b f\left(x_{3}\right) x_{4}+\varphi_{3}^{T} \hat{\theta}+\varphi_{3}^{T} \tilde{\theta}-y_{r}^{(3)}-\dot{\alpha}_{2} \\
& =b f\left(x_{3}\right)\left(z_{4}+\frac{\hat{\lambda}}{f\left(x_{3}\right)} y_{r}^{(3)}+\alpha_{3}\right)+\varphi_{3}^{T} \hat{\theta}+\varphi_{3}^{T} \tilde{\theta} \\
& -y_{r}^{(3)}-\dot{\alpha}_{2} \\
& =b f\left(x_{3}\right)\left(z_{4}+\frac{\hat{\lambda}}{f\left(x_{3}\right)} \bar{\alpha}_{3}\right)-b \tilde{\lambda} y_{r}^{(3)}+\varphi_{3}^{T} \hat{\theta}+\varphi_{3}^{T} \tilde{\theta} \\
& -\dot{\alpha}_{2} \\
& =b f\left(x_{3}\right) z_{4}-z_{2}-L_{3} z_{3}-\omega_{3}^{T} \hat{\theta}+\frac{\partial \alpha_{2}}{\partial \hat{\theta}} \Gamma \tau_{3} \\
& +\sum_{k=1}^{2}\left(\frac{\partial \alpha_{2}}{\partial x_{k}} x_{k+1}+\frac{\partial \alpha_{2}}{\partial y_{r}^{(k-1)}} y_{r}^{(k)}\right)-b \tilde{\lambda} \bar{\alpha}_{3} \\
& -b \tilde{\lambda} y_{r}^{(3)}+\varphi_{3}^{T} \hat{\theta}+\varphi_{3}^{T} \tilde{\theta}-\dot{\alpha}_{2} \\
& =-L_{3} z_{3}-z_{2}+\hat{b} f\left(x_{3}\right) z_{4}+\frac{\partial \alpha_{2}}{\partial x_{2}} \phi_{2}^{T} \hat{\theta} \\
& -b\left(\bar{\alpha}_{3}+y_{r}^{(3)}\right) \tilde{\lambda}+\omega_{3}^{T} \tilde{\theta}-\frac{\partial \alpha_{2}}{\partial x_{2}} \phi_{2}^{T} \hat{\theta}+\tilde{b} f\left(x_{3}\right) z_{4} \\
& +\frac{\partial \alpha_{2}}{\partial \hat{\theta}} \Gamma\left(\tau_{3}-\tau_{5}\right) \\
& =-L_{3} z_{3}-z_{2}+\hat{b} f\left(x_{3}\right) z_{4}+\sigma_{34} z_{4}+\sigma_{35} z_{5} \\
& \omega_{3}\left(\bar{\alpha}_{3}+y_{r}^{(3)}\right) \tilde{\lambda}+\tilde{b} f\left(x_{3}\right) z_{4} \\
& =b 0) \\
& =b
\end{aligned}
$$




$$
\begin{aligned}
& =z_{5}-\hat{b} z_{3}-L_{4} z_{4}-\omega_{4}^{T} \hat{\theta}+\frac{\partial \alpha_{3}}{\partial \hat{\theta}} \Gamma \tau_{4} \\
& +\sum_{k=1}^{2} \frac{\partial \alpha_{3}}{\partial x_{k}} x_{k+1}+f\left(x_{3}\right) \hat{b} \frac{\partial \alpha_{3}}{\partial x_{3}} x_{4}+\sum_{k=1}^{3} \frac{\partial \alpha_{3}}{\partial y_{r}^{(k-1)}} y_{r}^{(k)} \\
& -\hat{\lambda}\left(-\frac{b}{2 f\left(x_{3}\right)^{2}} x_{4}-\frac{\phi_{3}^{T} \hat{\theta}}{2 f\left(x_{3}\right)^{3}}-\frac{\phi_{3}^{T} \tilde{\theta}}{2 f\left(x_{3}\right)^{3}}\right) y_{r}^{(4)} \\
& -\dot{\alpha}_{4} \\
& =-L_{5} z_{5}-\sigma_{35} z_{3}-\sigma_{45} z_{4}-z_{4} \\
& +\frac{\partial \alpha_{3}}{\partial \hat{\lambda}} \dot{\hat{\lambda}}+\sum_{k=2}^{3} \frac{\partial \alpha_{k-1}}{\partial \hat{\theta}} \Gamma \omega_{4} z_{k}-\frac{\hat{\lambda} y_{r}^{(3)} \hat{b}}{2 f\left(x_{3}\right)^{2}} x_{4} \\
& -\hat{\lambda}\left(-\frac{b}{2 f\left(x_{3}\right)^{2}} x_{4}-\frac{\phi_{3}^{T} \hat{\theta}}{2 f\left(x_{3}\right)^{3}}-\frac{\phi_{3}^{T} \tilde{\theta}}{2 f\left(x_{3}\right)^{3}}\right) y_{r}^{(3)}-\dot{\alpha}_{3} \\
& =z_{5}-\hat{b} z_{3}-L_{4} z_{4}-\omega_{4}^{T} \hat{\theta}+\frac{\partial \alpha_{3}}{\partial \hat{\theta}} \Gamma\left(\tau_{4}-\tau_{5}\right) \\
& +\omega_{5}^{T} \tilde{\theta}+\tilde{b}\left(\frac{\hat{\lambda} y_{r}^{(4)}}{2 f\left(x_{3}\right)^{2}} x_{4}-f\left(x_{3}\right) \frac{\partial \alpha_{4}}{\partial x_{3}} x_{4}\right) \\
& \text { B. Lyapunov Derivative Including } \\
& \text { Valve Dynamics } \\
& -f\left(x_{3}\right) \tilde{b} \frac{\partial \alpha_{3}}{\partial x_{3}} x_{4}+\sum_{k=2}^{3} \frac{\partial \alpha_{k-1}}{\partial \hat{\theta}} \Gamma \omega_{4} z_{k} \\
& -\hat{\lambda}\left(-\frac{\phi_{3}^{T} \hat{\theta}}{2 f\left(x_{3}\right)^{3}}-\frac{\phi_{3}^{T} \tilde{\theta}}{2 f\left(x_{3}\right)^{3}}\right) y_{r}^{(3)}-\frac{\partial \alpha_{3}}{\partial x_{3}} \phi_{3}^{T} \hat{\theta} \\
& -\frac{\partial \alpha_{3}}{\partial x_{3}} \phi_{3}^{T} \tilde{\theta}-\frac{\partial \alpha_{3}}{\partial x_{2}} \phi_{2} \hat{\theta}-\frac{\partial \alpha_{3}}{\partial x_{2}} \phi_{2} \tilde{\theta}+\tilde{b} \frac{\hat{\lambda} y_{r}^{(3)}}{2 f\left(x_{3}\right)^{2}} x_{4} \\
& =-L_{4} z_{4}-\sigma_{34} z_{3}-\hat{b} z_{3}+z_{5}+\sigma_{45} z_{5} \\
& +\omega_{4}^{T} \tilde{\theta}+\tilde{b}\left(\frac{\hat{\lambda} y_{r}^{(3)}}{2 f\left(x_{3}\right)^{2}} x_{4}-f\left(x_{3}\right) \frac{\partial \alpha_{3}}{\partial x_{3}} x_{4}\right) \\
& \dot{z}_{5}=\dot{x}_{5}-\frac{d}{d t}\left(\frac{\hat{\lambda}}{f\left(x_{3}\right)} y_{r}^{(4)}\right)-\dot{\alpha}_{4} \\
& =u+\varphi_{5}^{T} \hat{\theta}+\varphi_{5}^{T} \tilde{\theta}-\frac{\dot{\hat{\lambda}}}{f\left(x_{3}\right)} y_{r}^{(4)}-\hat{\lambda} \frac{d}{d t}\left(\frac{1}{f\left(x_{3}\right)}\right) y_{r}^{(4)} \\
& -\frac{\hat{\lambda}}{f\left(x_{3}\right)} y_{r}^{(5)}-\dot{\alpha}_{4} \\
& =\alpha_{5}+\frac{\hat{\lambda}}{f\left(x_{3}\right)} y_{r}^{(5)}+\varphi_{5}^{T} \hat{\theta}+\varphi_{5}^{T} \tilde{\theta}-\frac{\dot{\hat{\lambda}}}{f\left(x_{3}\right)} y_{r}^{(4)} \\
& -\hat{\lambda}\left(-\frac{b}{2 f\left(x_{3}\right)^{2}} x_{4}-\frac{\phi_{3}^{T} \hat{\theta}}{2 f\left(x_{3}\right)^{3}}-\frac{\phi_{3}^{T} \tilde{\theta}}{2 f\left(x_{3}\right)^{3}}\right) y_{r}^{(4)} \\
& -\frac{\hat{\lambda}}{f\left(x_{3}\right)} y_{r}^{(5)}-\dot{\alpha}_{4} \\
& =-z_{4}-L_{5} z_{5}-\omega_{5}^{T} \hat{\theta}+\frac{\partial \alpha_{4}}{\partial \hat{\theta}} \Gamma \tau_{5} \\
& +\sum_{\substack{k=1 \\
k \neq 3}}^{4} \frac{\partial \alpha_{4}}{\partial x_{k}} x_{k+1}+f\left(x_{3}\right) \hat{b} \frac{\partial \alpha_{4}}{\partial x_{3}} x_{4}+\sum_{k=1}^{4} \frac{\partial \alpha_{4}}{\partial y_{r}^{(k-1)}} y_{r}^{(k)} \\
& +\left(\frac{y_{r}^{(4)}}{f\left(x_{3}\right)}+\frac{\partial \alpha_{4}}{\partial \hat{\lambda}}\right) \dot{\hat{\lambda}}+\sum_{k=2}^{4} \frac{\partial \alpha_{k-1}}{\partial \hat{\theta}} \Gamma \omega_{5} z_{k} \\
& +\frac{\partial \alpha 4}{\partial \hat{b}} \gamma \Pi_{5}-\frac{\hat{\lambda} y_{r}^{(4)} \hat{b}}{2 f\left(x_{3}\right)^{2}} x_{4}+\varphi_{5}^{T} \hat{\theta}+\varphi_{5}^{T} \tilde{\theta}-\frac{\dot{\hat{\lambda}}}{f\left(x_{3}\right)} y_{r}^{(4)} \\
& \dot{V}=z_{1}\left(-L_{1} z_{1}+z_{2}\right)+z_{2}\left(-L_{2} z_{2}-z_{1}+z_{3}+\omega_{2}^{T} \tilde{\theta}\right) \\
& +z_{3}\left(-L_{3} z_{3}-z_{2}+\hat{b} f\left(x_{3}\right) z_{4}+\sigma_{34} z_{4}+\sigma_{35} z_{5}\right. \\
& \left.+\omega_{3}^{T} \tilde{\theta}-b\left(\bar{\alpha}_{3}+y_{r}^{(3)}\right) \tilde{\lambda}+\tilde{b} f\left(x_{3}\right) z_{4}\right) \\
& +z_{4}\left(-L_{4} z_{4}-\sigma_{34} z_{3}-\hat{b} f\left(x_{3}\right) z_{3}+z_{5}+\sigma_{45} z_{5}\right. \\
& \left.+\omega_{4}^{T} \tilde{\theta}+\tilde{b}\left(\frac{\hat{\lambda} y_{r}^{(3)}}{2 f\left(x_{3}\right)^{2}} x_{4}-f\left(x_{3}\right) \frac{\partial \alpha_{3}}{\partial x_{3}} x_{4}\right)\right) \\
& +z_{5}\left(-L_{5} z_{5}-\sigma_{35} z_{3}-\sigma_{45} z_{4}-z_{4}\right. \\
& \left.+\omega_{5}^{T} \tilde{\theta}+\tilde{b}\left(\frac{\hat{\lambda} y_{r}^{(4)}}{2 f\left(x_{3}\right)^{2}} x_{4}-f\left(x_{3}\right) \frac{\partial \alpha_{4}}{\partial x_{3}} x_{4}\right)\right) \\
& +\tilde{\theta}^{T} \Gamma^{-1} \dot{\tilde{\theta}}+\frac{\tilde{b}}{\gamma} \dot{\tilde{b}}+\frac{|b|}{\gamma} \tilde{\lambda} \dot{\tilde{\lambda}} \\
& =-\sum_{k=1}^{5} L_{k} z_{k}^{2}+\left(\frac{\hat{\lambda} y_{r}^{(3)}}{2 f\left(x_{3}\right)^{2}} x_{4} z_{4}-f\left(x_{3}\right) \frac{\partial \alpha_{3}}{\partial x_{3}} x_{4} z_{4}\right. \\
& \left.+\frac{\hat{\lambda} y_{r}^{(4)}}{2 f\left(x_{3}\right)^{2}} x_{4} z_{5}-f\left(x_{3}\right) \frac{\partial \alpha_{4}}{\partial x_{3}} x_{4} z_{5}+f\left(x_{3}\right) z_{3} z_{4}-\frac{\dot{\hat{b}}}{\gamma}\right) \tilde{b} \\
& +\left(z_{2} \omega_{2}+z_{3} \omega_{3}+z_{4} \omega_{4}+z_{5} \omega_{5}-\Gamma^{-1} \dot{\hat{\theta}}\right)^{T} \tilde{\theta} \\
& -\left(b\left(\bar{\alpha}_{3}+y_{r}^{(3)}\right) z_{3}-\frac{|b|}{\gamma} \dot{\hat{\lambda}}\right) \tilde{\lambda} \\
& =-\sum_{k=1}^{5} L_{k} z_{k}^{2}
\end{aligned}
$$

